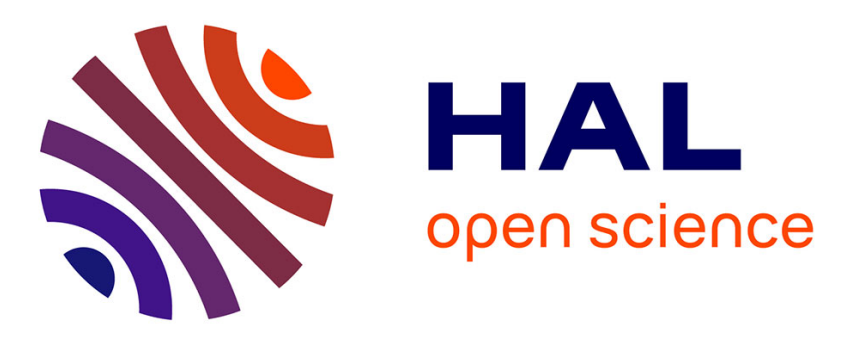

\title{
Discussion about sliding mode algorithms, Zeno phenomena and observability
}

Lei Yu, Jean-Pierre Barbot, Djamila Benmerzouk, Driss Boutat, Thierry Floquet, Gang Zheng

\section{To cite this version:}

Lei Yu, Jean-Pierre Barbot, Djamila Benmerzouk, Driss Boutat, Thierry Floquet, et al.. Discussion about sliding mode algorithms, Zeno phenomena and observability. Fridman, Leonid; Moreno, Jaime; Iriarte, Rafael (Eds.). Lecture Notes in Control and Information Sciences, 2012, Volume 412, Springer, 2012. hal-00750621

\section{HAL Id: hal-00750621 \\ https://hal.inria.fr/hal-00750621}

Submitted on 11 Nov 2012

HAL is a multi-disciplinary open access archive for the deposit and dissemination of scientific research documents, whether they are published or not. The documents may come from teaching and research institutions in France or abroad, or from public or private research centers.
L'archive ouverte pluridisciplinaire HAL, est destinée au dépôt et à la diffusion de documents scientifiques de niveau recherche, publiés ou non, émanant des établissements d'enseignement et de recherche français ou étrangers, des laboratoires publics ou privés. 


\title{
Discussion about sliding mode algorithms, Zeno phenomena and observability
}

L. Yu, J-P Barbot, D. Benmerzouk, D. Boutat, T. Floquet and G. Zheng

\begin{abstract}
This chapter is devoted to a discussion about the relations between first and high order sliding mode algorithms and both types of Zeno (Chattering and Genuinely) behaviors of switched dynamical systems. Firstly, the Henstock-Kurzweil integral is recalled in order to set up the problem of switched systems with Zeno phenomena, which enables to include Filippov solution and take into account some singularities. Then, observer designs based on the well-known super twisting algorithm are proposed. For this kind of problems, the importance of finite time convergence of the observation error is studied, and some simulations are given to highlight the discussion. Lastly, the two tanks example is given in order to point out the differences between both Zeno phenomena types, to show that there is life after Zeno and that a higher order sliding mode observer can be efficient before, during and after both Zeno phenomena types.
\end{abstract}

\section{Yu}

Signal Processing Laboratory, Electronic and Information School, Wuhan University, China, and ECS ENSEA, 6 Avenue du Ponceau, 95014 Cergy-Pontoise, France e-mail: lei.yu @ensea.fr

J-P Barbot

ECS ENSEA, 6 Avenue du Ponceau, 95014 Cergy-Pontoise, France and Project Non-A, INRIA Lille - Nord Europe e-mail: barbot@ensea.fr

D. Benmerzouk

Department of Mathematics, Tlemcen University, Algeria, e-mail: d_benmerzouk@yahoo.fr

D. Boutat

PRISME ENSIB, 10 Boulevard de Lahitolle, 18020 Bourges, Fraance e-mail: driss.boutat@ensibourges.fr

T. Floquet

LAGIS, UMR FRE 3303, Ecole Centrale de Lille, Cité Scientifique, BP 48, 59651 Villeneuved'Ascq Cedex, France and Project ALIEN, INRIA Lille - Nord Europe e-mail: thierry.floquet@eclille.fr

G. Zheng

Project Non-A, INRIA Lille - Nord Europe, 40, avenue Halley, 59650 Villeneuve d'Ascq, France e-mail: gang.zheng@inria.fr 
This paper is a partial draft of the chapter in Lecture Notes in Control and Information Sciences, 2012, Volume 412

\section{Discussion on Zeno and sliding mode behavior}

First order sliding mode concept has been well-known in control system theory for at least fifty years, especially since the book of Filippov [14]. A system exhibiting a first order sliding mode behavior can be seen as a variable structure system converging in finite time towards some constraint manifold and then sliding on this manifold by means of permanent switches at an infinite frequency between two system structures. Commutations at infinite frequency between two subsystems is named as Zeno ${ }^{1}$ phenomena in the hybrid dynamical system theory. The relation between first order sliding mode and Zeno phenomena generates a huge set of questions, such as:

1. Is first order sliding mode the only Zeno phenomena?

2. Do all Zeno phenomena always exhibit a sliding mode?

3. Is it possible to use a sliding mode approach in the context of hybrid dynamical systems?

4. Is there life after Zeno?

5. Are specific mathematical tools required?

The answer to the first question is no. In fact, since at least the pioneer works of S. Emelyanov, S. Korovin and A. Levant [13], [26], a new type of sliding modes has been introduced called higher order sliding modes. This type of sliding mode ensures a finite time convergence onto a constraint manifold defined by the vanishing of some constraint variable $s$ and at least its first derivative $\dot{s}$ along the system trajectories. The requirement that $\dot{s}$ must be also equal to zero in finite time has an important consequence on the qualitative behavior of Zeno phenomena. For example, in [31] Chapter 8 page 228, it is proved that the twisting algorithm generates an infinite number of commutation in finite time, where any dwell time ${ }^{2}$ is strictly different to zero. Actually, one can distinguish different qualitative behaviors such as the chattering Zeno phenomena and the genuinely Zeno phenomena, see Ames et al. [2]. Roughly speaking, the chattering Zeno phenomena is equivalent to obtain a dwell time equal to zero after a finite number of commutations, as it is exactly the case for first order sliding mode. On the other hand, the genuinely Zeno phenomena corresponds to obtain an infinity of commutation before obtaining a dwell time equal to zero, and this is exactly the case of the twisting algorithm.

The answer to the second question is also no. For instance, consider the bouncing ball example (see [2] and [20]). This is a switched system with jumps (actually an impact system [6]) that exhibits a Zeno phenomena. This kind of system has only one dynamic and has a jump state at the impact instant before to roll on the floor. It

${ }^{1}$ This refers to Zeno of Elea and its paradoxes, see for example "Achille and the tortoise"

2 time interval between two switching instants 
is shown in [2] that the bouncing ball case generates a genuinely Zeno phenomena and that the solution after the Zeno point (impact accumulation point) satisfies a holonomic constraint which is different from the Filippov solution.

The answer to the third question is yes. This will be proved in Section 4 where a sliding mode observer for a particular class of hybrid systems will be designed on the basis of our previous work [34].

The answer to the fourth question is also yes. In the example of the bouncing ball, even if the model changes after the Zeno time, meaning in some sense that "there is no life of the original model after Zeno", it will be shown that it is not the case for the two tanks example.

The answer to the fifth question is also yes. In fact, in order to consider the most general class of systems, the most general definition of integral has to be considered. To the best of our knowledge, the most general integral definition is the HenstockKurzweil-Pettis integral (H-K-P integral). Nevertheless, for the sake of simplicity, this chapter will only deal with the Henstock-Kurzweil integral (H-K integral) [18] which is recalled in the Section 3.

\section{Zeno Types}

In this chapter, we will consider Hybrid Dynamical Systems belonging to the following class of switched systems without state jump:

$$
\left\{\begin{array}{l}
\dot{x}=f(x)+g(x) q=f(x)+\sum_{i=1}^{n} g_{i}(x) q_{i} \\
y=h(x)
\end{array}\right.
$$

where $x \in U \subset R^{n}$ is the set of admissible continuous state, $y \in R$ is the measured output and where the vector fields $f: U \rightarrow R^{n}, g_{i}: U \rightarrow R^{n}$ and $h: U \rightarrow R$ are sufficiently smooth. Moreover $q_{i}: R^{+} \rightarrow Q=\{0,1\}$ is the discrete component of the discrete vector $q$.

The definition of time trajectory and Zeno phenomenon given in $[28,23]$ can be stated as follows:

Definition 1. A time trajectory is a finite or infinite sequence of intervals $T_{N}=$ $\left\{I_{i}\right\}_{i=0}^{N}$, where $N \in \mathbb{N}$, such that:

- $I_{i}=\left[\tau_{i}, \tau_{i+1}\right], \tau_{i} \leq \tau_{i+1}$ for all $0 \leq i \leq N$.

- If $N$ is limited, either $I_{N}=\left[\tau_{N}, \tau_{N+1}\right]$ with $\tau_{N} \leq \tau_{N+1}$ and $\tau_{\infty}$ bounded or $I_{N}=$ $\left[\tau_{N}, \tau_{N+1}\left[\right.\right.$ for $\tau_{N} \leq \tau_{N+1} \leq \infty$.

- For all $i$ corresponds a discrete transition $q_{i}(t)$ such that $q_{i}(t)$ is constant for $t \in\left[\tau_{i}, \tau_{i+1}\right]$.

- For all $i$ and $t \in\left[\tau_{i}, \tau_{i+1}\right]$ corresponds a continuous evolution $x(t)$. 


\section{Definition 2.}

A dynamical system is called Zeno if $t_{\infty}:=\sum_{i=0}^{\infty}\left(\tau_{i+1}-\tau_{i}\right)$ is bounded.

This means that the system takes an infinite amount of discrete transitions in a finite amount of time, in this case the time $t_{\infty}$ is called Zeno time.

It is known that there exist two fundamental types of Zeno:

\section{Definition 3.}

- Chattering Zeno: There exist a finite number $C \in \mathbb{N}$ and a bounded value $t_{\infty}$ such that:

$$
t_{\infty}=\sum_{i=0}^{\infty}\left(\tau_{i+1}-\tau_{i}\right) \quad \text { and } \quad \forall j>C, \quad\left[t_{j+1}-t_{j}\right]=0 .
$$

- Genuinely Zeno: There exist $C \in \mathbb{N}$ and a bounded value $t_{\infty}$ such that:

$$
t_{\infty}=\sum_{i=0}^{\infty}\left(\tau_{i+1}-\tau_{i}\right) \quad \text { and } \quad \forall j>C, \quad\left[t_{j+1}-t_{j}\right]>0 .
$$

Consider for example the twisting algorithm $[13,17,26]$. It is clear that the convergence on the constrained manifold given by $s=\dot{s}=0$ is due to a Genuinely Zeno phenomenon [31]. Nevertheless, after the finite time convergence on this manifold, the Zeno behavior is a chattering phenomenon. So, for the twisting algorithm the Zeno type changes but the process never leaves the Zeno behavior.

\section{Mathematical recalls of $\mathrm{H}-\mathrm{K}$ integral}

The H-K integral, also known as the gauge integral, the generalized Riemann integral, was defined independently by Henstock and Kurzweil in the 1950's. Let us recall both the definitions related to the Riemann integral and the $\mathrm{H}-\mathrm{K}$ integral in order to clarify their differences.

Definition 1 Consider a real function $f$ defined on an interval $[a, b] \subset R$. For any tagged partition $P$ of $[a, b]$ such as $a=x_{0}<x_{1}<x_{2}<\cdots<x_{n}=b$, and $\tau_{i} \in\left[x_{i-1}, x_{i}\right] \subset[a, b]$, consider

$$
f(P):=\sum_{i=1}^{n} f\left(\tau_{i}\right)\left(x_{i}-x_{i-1}\right) .
$$

For any given function $\delta:[a, b] \rightarrow R_{+}^{*}, P$ is said to be $a$-fine tagged partition of $[a, b]$ if $h_{i}:=x_{i}-x_{i-1}<\delta\left(\tau_{i}\right)$ where $\delta$ is called a gauge for each $i$.

Definition 2 A number I is called the Riemann integral (respectively the $H$-K integral) of $f:[a, b] \rightarrow R$ if for each constant $\varepsilon>0$, there exists a constant $\delta$ (respectively a function $\left.\delta:[a, b] \rightarrow R_{+}^{*}\right)$, such that whenever $P$ is a $\delta$-fine tagged partition of $[a, b]$, one has $|I-f(P)|<\varepsilon$.

Roughly speaking, the integral of $f$ on $[a, b]$ is obtained by approximating the region under the curve defined on $[a, b]$ as a union of small rectangles. The Riemann 
integral requires that all those rectangles depend on a constant $\delta$ while the H-K integral uses a more sophisticated $\delta$ ( $\delta$ depends on $\tau_{i}$ ). In fact, when a function $f$ oscillates more quickly at some points of the interval $[a, b]$, one has to tighten the step $h_{i}$ at these points in order to approximate more accurately the associated surface. This is possible by choosing $h_{i}<\delta\left(\tau_{i}\right)$ such that $\delta\left(\tau_{i}\right)$ is a sufficiently small positive value that depends on the place from where the rectangle of height $f\left(\tau_{i}\right)$ is considered (see Fig. 1).

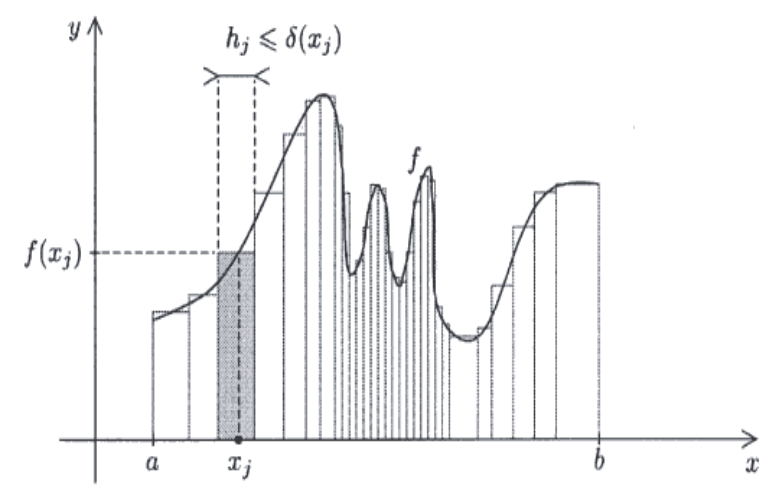

Fig. 1 Illustrative explanation of H-K Gauge

To highlight the principle of the H-K integral, consider the two following examples.

Example 1 Consider the function $f$ defined on $[0,1]$ as follows:

$$
f(x)= \begin{cases}x^{-1} \sin \left(x^{-2}\right) & \text { on }] 0,1] \\ 0 & \text { for } x=0 .\end{cases}
$$

This function is neither Riemann nor Lebesgue integrable, but H-K integrable, and this can be proved by using a special choice of gauge (that requires skill and effort) given as follows:

$$
\delta(\tau)= \begin{cases}\sqrt{\varepsilon} & \text { if } \tau=0 \\ \min \left\{\frac{\tau}{2}, \frac{\varepsilon \tau^{4}}{24}\right\} & \text { if } 0<\tau \leq 1 .\end{cases}
$$

Example 2 Consider the function $g$ defined on [0, 1] as follows:

$$
g(x)= \begin{cases}x & \text { if } x \in \mathscr{Q} \\ 0 & \text { if } x \notin \mathscr{Q}\end{cases}
$$

where $\mathscr{Q}$ represents the set of rationales. This function is discontinuous everywhere, and thus it is not Riemann integrable but $H$-K integrable. It can be proved that its value is equal to 0 by using a special choice of the gauge given by: 


$$
\delta(\tau)= \begin{cases}2^{-j-1} \varepsilon & \text { if } \tau=p_{j} \\ 1 & \text { if } \tau \notin \mathscr{Q}\end{cases}
$$

where $p_{1}, p_{2}, \ldots, p_{j}, \ldots$ are the rationales.

Thus, taking a more general form of the gauge $\delta$ (instead of $\delta$ constant) yields a richer class of possible integrands and permits to obtain that:

Riemann integrable functions $\subset$ Lebesgue integrable functions $\subset \mathrm{H}-\mathrm{K}$ integrable functions

Note that the H-K integral can also be generalized to bounded or unbounded sets in $R^{n}$ and to more general spaces such as Banach spaces or ordered spaces. The most important feature of the H-K integral is its wider applicability. Moreover, it can avoid abstract notions such as set theory, $\sigma$ algebras or inner and outer measures. This can be seen from the equivalence:

a set $E \subset[a, b]$ is Lebesgue integrable $\Longleftrightarrow$ its characteristic function is $H-K$ integrable

Moreover, many classical results formulated in the framework of the Lebesgue theory, such as the Lemma of Fatou or the fundamental theorem of calculus, can be simplified (assumptions on continuity for example are omitted when H-K integrability is considered) [18].

Another important result is the Hake's Theorem which states that it is not necessary to consider "improper" H-K integral (as it is the case of Riemann integral) because the construction of the H-K integral makes any "improper" $\mathrm{H}-\mathrm{K}$ integral be a "proper" integral. Note also that other integrals, such as Denjoy integral, Perron integral, Lee and Vyborny integral, all turn out to be "equivalent" to the H-K integral.

Some authors proposed an extension of the H-K integral, called KurzweilHenstock-Pettis integral (H-K-P integral) (see [8]) which offers an interesting possible applicability to Fourier analysis and differential equations. In this case, the solution of a Cauchy problem given by:

$$
\dot{X}=f(t, X(t)) \text { with } X(0)=X_{0}
$$

is studied as the solution of the following integral equation:

$$
X(t)=X_{0}+\int_{0}^{t} f(\mu, X(\mu)) d \mu
$$

where the integral is in the sense of H-K. It does not require strong assumptions on the considered system. So it can be seen as a tool that defines more accurately (and take into account) the specific and quick oscillations of the considered dynamics. 


\section{Observability and observer design for some classes of Hybrid dynamical system}

The following three classes of switched systems without jump are considered.

\subsection{First basic observability form}

The first proposed observability form is:

$$
\begin{aligned}
\left(\begin{array}{c}
\dot{z}_{1} \\
\dot{z}_{2} \\
\dot{z}_{3} \\
\vdots \\
\dot{z}_{n}
\end{array}\right) & =\left(\begin{array}{ccccc}
0 & \cdots & 0 & 0 & 0 \\
1 & \ddots & 0 & 0 & 0 \\
0 & \ddots & 0 & 0 & 0 \\
\vdots & \ddots & \ddots & \ddots & \vdots \\
0 & \cdots & 0 & 1 & 0
\end{array}\right)\left(\begin{array}{c}
z_{1} \\
z_{2} \\
z_{3} \\
\vdots \\
z_{n}
\end{array}\right)+\left(\begin{array}{c}
\tilde{f}_{q} \\
0 \\
0 \\
\vdots \\
0
\end{array}\right) \\
y & =\tilde{h}(z)=z_{n}
\end{aligned}
$$

where $q$ is the discrete vector and $\tilde{f}_{q}$ is a continuous function with respect to $z$ and parameterized by $q$. This system is regularly weakly locally observable and the observability is independent from the discrete vector $q$.

\subsection{Second basic observability form}

The second proposed observability form is:

$$
\begin{aligned}
& \dot{z}=\alpha_{0}(z)+\gamma_{q}(y), \quad q \in Q \\
& y=\tilde{h}(z)=z_{n}
\end{aligned}
$$

where $z \in \mathbb{R}^{n}$ and $y \in \mathbb{R}$

Assumption 1 The pair $\left(\alpha_{0}(z), y\right)$ is regularly locally weakly observable ${ }^{3}$.

\footnotetext{
${ }^{3}$ In addition to the classical locally weakly observability condition (see [19]), the first $n-1$ derivatives in the rank condition are requested to be regular i.e.

$$
\operatorname{Rank}\left(\begin{array}{c}
d \tilde{h} \\
\vdots \\
d L_{\alpha_{0}}^{n-1} \tilde{h}
\end{array}\right) \mid z=n
$$
}




\subsection{Extended observability form}

The following extended observability form includes both previous forms:

$$
\begin{aligned}
\left(\begin{array}{c}
\dot{\xi}_{1} \\
\vdots \\
\dot{\xi}_{r-1}
\end{array}\right) & =\left(\begin{array}{ccccc}
0 & 1 & 0 & \ldots & 0 \\
0 & 0 & 1 & \ldots & 0 \\
\vdots & \vdots & \vdots & \ddots & \vdots \\
0 & 0 & 0 & \ldots & 1
\end{array}\right)\left(\begin{array}{c}
\xi_{1} \\
\vdots \\
\xi_{r-1} \\
\xi_{r}
\end{array}\right) \\
\dot{\eta}_{1}=\dot{\xi}_{r} & =\alpha_{0}(\xi, \eta)+\gamma_{q}(\xi) \\
\dot{\tilde{\eta}} & =\beta_{0}(\xi, \eta)+\delta_{q}(\xi) \\
y & =\xi_{1}
\end{aligned}
$$

where $\xi=\left(\xi_{1} \cdots \xi_{r}\right)^{T}, \eta=\left(\eta_{1}, \cdots, \eta_{n-r+1}\right)^{T}$ and $\tilde{\eta}=\left(\eta_{2}, \cdots, \eta_{n-r+1}\right)^{T}$.

\section{Assumption 2}

The pair $\left(\left(\begin{array}{c}\alpha_{0}(\xi, \eta) \\ \beta_{0}(\xi, \eta)\end{array}\right), \eta_{1}\right)$ is uniformly weakly locally observable . $^{4}$

In this paper, any consideration on the existence of the diffeomorphisms which transform a switched system into one of these forms is given, nevertheless sufficient conditions may be found in our previous work [34].

\subsection{Discussion on the observability of the first basic observability form}

For the form (2)-(3), an estimate of the continuous state $\xi$ can be obtained via an algebraic estimator as defined in [29, 30], a sliding mode observer $([3,12])$, or a high order sliding mode observer $[9,16,27,32]$, because $y$ and the $n-1$ first time derivatives of $y$ provide sufficient information in order to estimate the continuous state.

\subsection{Discussion on the observability of the second basic observability Form}

In the form (4)-(5), the discrete state $q$ is not considered as an unknown perturbation but as an input, consequently the following assumption is requested:

${ }^{4}$ uniformly with respect to $\xi$. 


\section{Assumption 3}

The discrete state $q$ is known and $\gamma_{q}(y)$ is Henstock-Kurzweil-Pettis integrable ${ }^{5}$ ( see [18], [8], [33]) or NV-Integrable [10].

Clearly, this assumption can not be satisfied in the case of Zeno phenomena, such as for the Chattering Zeno (i.e. after some time the dwell time is exactly equal to zero) or for the Genuinely Zeno (i.e. the dwell time is never equal to zero) [2]. So, in practice, it is only possible to obtain the filtered discrete state $q_{f}$, instead of the real discrete state $q$. Consequently, Assumption 3 is replaced by the following one:

\section{Assumption 4}

- $a$-The system (4)-(5) is affine with respect to the discrete state q, i.e. $\gamma_{q}(y)=$ $\gamma(y) q$, where $\gamma$ is at least a $C^{1}$ function of $y$.

- $\quad b$ - The functions $\gamma(y) q$ and $\gamma(y) q_{f}$ are Henstock-Kurzweil-Pettis integrable and the mean value $q_{f}$ is measured via a low pass filter with a large enough bandwidth on the time interval $[0, \alpha]$.

Moreover, consider the following system:

$$
\begin{aligned}
& \dot{z}=\alpha_{0}(z) \\
& y=\tilde{h}(z)=z_{n}
\end{aligned}
$$

and assume that:

Assumption 5 For the system (10)-(11), there exists an observer such that the continuous state observation error (i.e. the difference between the continuous state ant its estimate) is exponentially stable.

Remark 1 Under some specific assumptions such as Lipschitz condition or persistent excitation, it is possible to design classical high gain observers [4] or adaptive observers [7]. Without the perturbation term, those observers can guarantee the exponential stability of the continuous state observation error.

Then, it is possible to set the following proposition:

\section{Proposition 1}

-A) Under Assumptions 1, 3 and 5, system (4)-(5) with Zeno phenomenon is observable 6 .

-B) Under Assumptions 1, 4 and 5, system (4)-(5) with Zeno phenomenon is practically observable ${ }^{7}$.

\footnotetext{
5 see also Denjoy-Khinchine integrable.

${ }^{6}$ The observation error can be assigned to zero

${ }^{7}$ The observation error can be constrained to lie within any measurable vicinity of zero but not zero.
} 
Proof. Proof of part $A$ : there exists an observer of the form:

$$
\begin{aligned}
& \dot{\hat{z}}=\beta(\hat{z}, y, \hat{y}) \\
& \hat{y}=\hat{z}_{n}
\end{aligned}
$$

which ensures the exponential stability of the continuous state observation error $e=z-\hat{z}$ for system (10), (11). Consequently, there exists a Lyapunov function $V(e)$ with respect to (10), (11) and (12), (13) such that:

$$
\dot{V}=\frac{\partial V}{\partial e}\left(\alpha_{0}(z)-\beta(\hat{z}, y, \hat{y})\right)<-K V
$$

with $K>0$.

Modifying (12), (13) as follows:

$$
\begin{aligned}
& \dot{\hat{z}}=\beta(\hat{z}, y, \hat{y})+\gamma_{q_{f}}(y) \quad \text { with } \gamma_{q_{f}}(y)=\gamma(y) q_{f} \\
& \hat{y}=z_{n}
\end{aligned}
$$

the state observation error for system (4)-(5) and observer (15)-(16) is exponentially stable. Using the previous Lyapunov function, one has:

$$
\dot{V}=\frac{\partial V}{\partial e}\left(\alpha_{0}(z)-\beta(\hat{z}, y, \hat{y})+\gamma_{q}(y)-\gamma_{q_{f}}(y)\right)<-K^{\prime} V
$$

where $K^{\prime}=K+\sup _{t \in[0, \alpha]}\{|o(y(t))|\}$.

Proof of part $B$ : the observation error for systems (4)-(5) and (15)-(16) becomes:

$$
\dot{e}=\alpha_{0}(z)-\beta(\hat{z}, y, \hat{y})+\gamma(y)\left(q-q_{f}\right)
$$

From condition b) of Assumption 4, one obtains that

$$
\dot{p}=\gamma(y) p \quad \text { with } \quad p=q-q_{f}
$$

is a Cauchy problem for each fixed initial value $p_{0}$ in the sense of HenstockKurzweil-Pettis integrals.

Using the same method as Filippov in [15] page 17 and setting $\varepsilon=e-p$, one has:

$$
\dot{\varepsilon}=\alpha_{0}(z)-\beta(\hat{z}, y, \hat{y})
$$

which admits a local solution in the Carathéodory sense for $t \in[0, \Gamma] \subset[0, \alpha]$. Moreover, Assumption 5 and the observer (12)-(13) ensure that there exists a Lyapunov function $V(e)$ for (10)-(11) which satisfies (14). Consequently, derivation of $V(\varepsilon)$ with respect to (17) gives:

$$
\dot{V}=\frac{\partial V}{\partial \varepsilon}\left(\alpha_{0}(z)-\beta(\hat{z}, y, \hat{y})\right)
$$


Since $\varepsilon=e-p$, one has $\left.\frac{\partial V}{\partial \varepsilon}\right|_{\varepsilon}=\left.\frac{\partial V}{\partial e}\right|_{\varepsilon}$ and from Assumption 5, it is possible to rewrite (18) as follows:

$$
\begin{aligned}
\dot{V}= & \left.\frac{\partial V}{\partial e}\right|_{e}\left(\alpha_{0}(z)-\beta(\hat{z}, y, \hat{y})\right) \\
& -\left.\frac{1}{2} \frac{\partial^{2} V}{\partial e^{2}}\right|_{e}\left[O(p) \otimes\left(\alpha_{0}(z)-\beta(\hat{z}, y, \hat{y})\right)\right]+O\left(p^{2}\right)
\end{aligned}
$$

with $\lim _{p \rightarrow 0} O(p)=0$.

As for all $\varepsilon>0$, there exists a filter and $t_{1} \geq 0$ such that $\forall t>t_{1}$, we have

$$
\|p\|=\sup _{0 \leq t \leq \Gamma}\left|\int_{t_{1}}^{t} \gamma(y(\sigma))\left(q(\sigma)-q_{f}(\sigma)\right) d \sigma\right|<\varepsilon
$$

one obtains

$$
\begin{aligned}
\dot{V}(\varepsilon) & \leq-K^{\prime} V(\varepsilon)+\frac{1}{2}\left|\frac{\partial^{2} V}{\partial e^{2}}\right|_{e}\left[O(p) \otimes\left(\alpha_{0}(z)-\beta(\hat{z}, y, \hat{y})\right)\right] \mid \\
& +\left|O\left(\|p\|^{2}\right)\right|
\end{aligned}
$$

Consequently, it is possible, for each $V_{d}>0$, to set $\varepsilon<<\frac{V_{d}}{2}$ and the inequality (19) becomes

$$
\dot{V}(\varepsilon) \leq-K^{\prime} V(\varepsilon)+|O(\varepsilon)|
$$

So, one can conclude that for $e \notin E_{V_{d}}:=\left\{e: V(e)<V_{d}\right\}$, one has:

$$
\dot{V}(\varepsilon) \leq-\frac{K^{\prime \prime}}{2} V(\varepsilon) \quad \text { for some } \quad K^{\prime \prime}>0
$$

because $\varepsilon<<\frac{V_{d}}{2}$ guarantees that $|O(\varepsilon)|<\frac{V_{d}}{2}$.

\subsection{Observability for the extended observability Form}

For system (6)-(9), according to the previous discussion on the observability for the two basic forms, one can easily conclude:

\section{Corollary 1}

Under Assumptions 2, 4 and 5, the system (6)-(9) with Zeno phenomenon is practically observable.

Consequently, it is possible to design an hybrid observer for this system: for the observation of $\xi$, a step-by-step high order sliding mode observer can be designed to obtain a finite time estimate of $\xi$. So, after the convergence of $\xi$, $\xi_{r}$ (or $\eta_{1}$ ) can be considered as an output, and one can design an asymptotic observer (for example a high gain observer) to estimate $\eta$, as shown in Figure 2. 


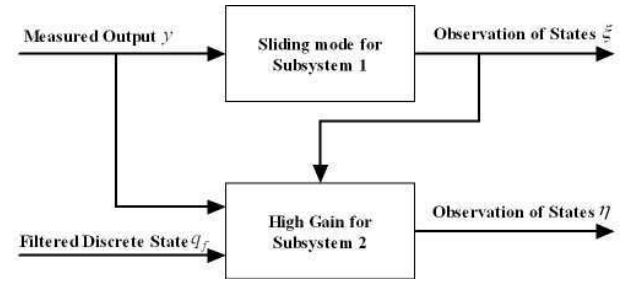

Fig. 2 Hybrid observer scheme.

Example 3 Consider the following system:

$$
\left\{\begin{array}{l}
\dot{z}_{1}=z_{2} \\
\dot{z}_{2}=z_{3}-z_{2}^{3}+\frac{3}{2} z_{2}+\frac{1}{2} \gamma_{q}\left(z_{2}\right) \\
\dot{z}_{3}=-z_{2} \\
y=z_{1}
\end{array}\right.
$$

with $\gamma_{q}\left(z_{2}\right)=\operatorname{sign}(w) \cdot z_{2}$, where $w$ is a high frequency noise. This form of $q$ theoretically leads to a Zeno phenomena.

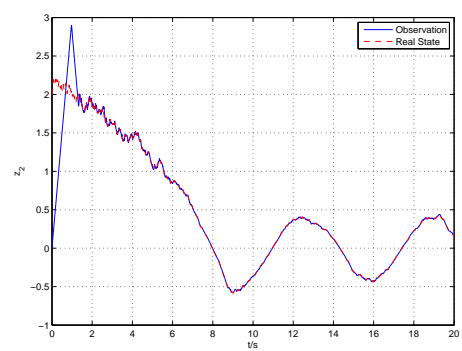

Fig. 3 Results of Observer for system (20) for $z_{2}$.

For this example, one can follow the scheme shown in Fig. 2 to build an hybrid observer. First, one considers the states $z_{1}$ and $z_{2}$, where $z_{1}$ is known and $z_{2}$ is observed through a sliding mode observer. Denote $\hat{z}_{2}$ as the observation of $z_{2}$. Then the remaining state dynamics has the following form:

$$
\left\{\begin{array}{l}
\dot{z}_{2}=z_{3}-z_{2}^{3}+\frac{3}{2} z_{2}+\frac{1}{2} \gamma_{q}\left(z_{2}\right) \\
\dot{z}_{3}=-z_{2} \\
y^{\prime}=z_{2}
\end{array}\right.
$$




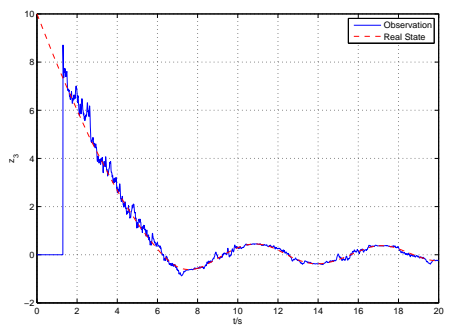

Fig. 4 Results of Observer for system (20) for $z_{3}$.

where the value of $z_{2}$ is taken as the output of the subsystem (21), i.e. $y^{\prime}=\hat{z}_{2}$. One can observe the state $z_{3}$ using a high gain observer. The performances are shown in Fig. 3 and 4, where Fig. 3 shows the state $z_{2}$ and the observed state $\hat{z}_{2}$ and where Fig. 4 depicts the same for $z_{3}$. In fact, the high gain observer (with $\hat{z}_{2}$ as output and $z_{2}, z_{3}$ as states) must be switched on only after the convergence of the finite time sliding mode observer (with $z_{1}$ as output and $z_{1}, z_{2}$ as states). Hence one can assign $\hat{z}_{3}$ to be zero at the beginning of the observation procedure.

\section{The two tanks example}

consider the two water tanks example [21] in Fig. 5, which is a typical switched system where Zeno behavior may exist if the commutation of the water distribution between both tanks is considered instantaneous with respect to the other dynamics.

The simplified model of the two water tanks system described in Fig. 5 is given by:

$$
\left(\begin{array}{l}
\dot{h}_{1} \\
\dot{h}_{2}
\end{array}\right)=\left(\begin{array}{c}
v-v_{1} \\
-v_{2}
\end{array}\right)+\left(\begin{array}{c}
-v \\
v
\end{array}\right) q
$$

where $h_{1}, h_{2}$ are the water levels for each tanks, $v_{1}, v_{2}$ are the flows of water out of the tanks, $v$ is a constant input flow of water which goes through a pipe and into either tank at any particular time point, and $q$ is the switching state of the pipe which is determined by the transient conditions (see Fig. 6 and Fig. 7 for two different cases). By considering Torricelli's law, one has $v_{1}=\sqrt{2 g h_{1}}$ and $v_{2}=\sqrt{2 g h_{2}}$, where $g$ is the gravity. Then the dynamic can be rewritten as

$$
\left(\begin{array}{c}
\dot{h}_{1} \\
\dot{h}_{2}
\end{array}\right)=\left(\begin{array}{c}
v-\sqrt{2 g h_{1}} \\
-\sqrt{2 g h_{2}}
\end{array}\right)+\left(\begin{array}{c}
-v \\
v
\end{array}\right) q
$$

Assume that the only measured signal is $y=h_{1}+h_{2}$ ( $v$ and $q$ are supposed to be unknown). Then the system is regularly locally weakly observable: 


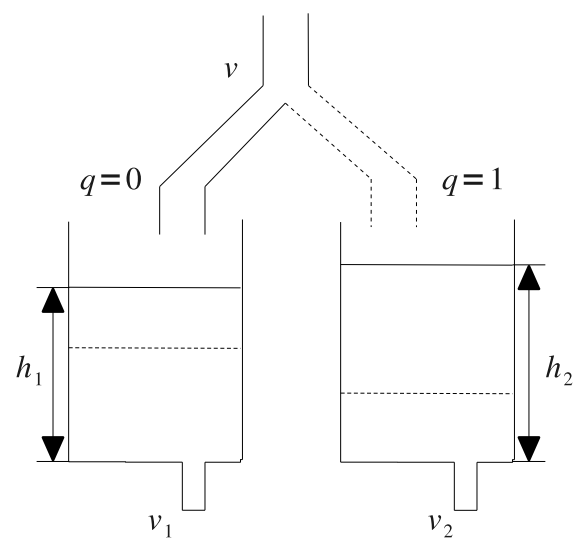

Fig. 5 Two water tanks system

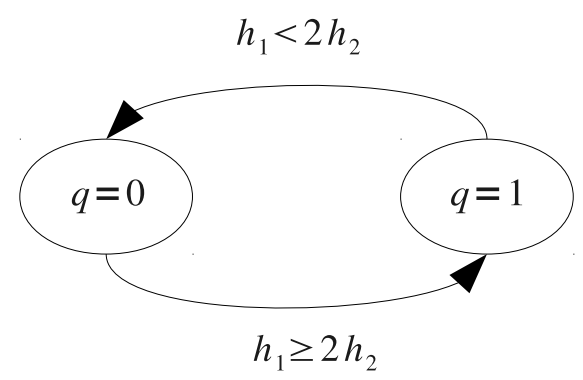

Fig. 6 The transient of discrete state for the case $1\left(h_{1}=2 h_{2}\right)$.

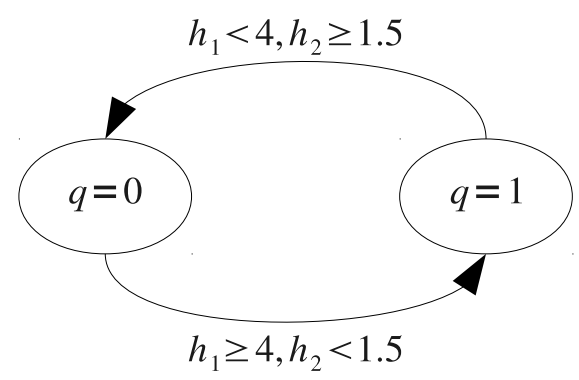

Fig. 7 The transient of discrete state for the case $2\left(h_{1}=4\right.$ and $\left.h_{2}=1.5\right)$.

$$
\operatorname{rank}\left(\begin{array}{c}
d y \\
d \dot{y}
\end{array}\right)=\operatorname{rank}\left(\begin{array}{cc}
1 & 1 \\
\frac{-1}{\sqrt{2 g h_{1}}} & \frac{-1}{\sqrt{2 g h_{2}}}
\end{array}\right)=2
$$


excepted on the set $S_{O}$

$$
S_{O}=\left\{\left(h_{1}, h_{2}\right)^{T}: h_{1}=h_{2}\right\} .
$$

This set is a set of observability singularities and it is a manifold of dimension $n-1=1$. Moreover, $S_{O}$ separates the state space in two parts. It is important to note that $S_{O}$ characterizes the singularity observability considering only $y$ and $\dot{y}$. However, there exist several different observability definitions for nonlinear systems and hybrid systems [5], [11], [20], [22],[25], involving high order output derivatives with order greater than the dimension of the state space.

Moreover, the system satisfies the observability matching condition [31] ${ }^{8}$

$$
\frac{\partial y}{\partial q}=\frac{\partial \dot{y}}{\partial q}=0
$$

Conditions (22) and (23) are necessary and sufficient conditions for the existence of a diffeomorphism which transforms, at least locally, the system (21) into the first basic normal form. Then, under the diffeomorphism $\xi=\phi\left(h_{1}, h_{2}\right)$ with $\phi_{1}\left(h_{1}, h_{2}\right)=$ $h_{1}+h_{2}$ and $\phi_{2}\left(h_{1}, h_{2}\right)=v-\sqrt{2 g h_{1}}-\sqrt{2 g h_{2}}$, the system is locally transformed into the first basic normal form (2)-(3):

$$
\begin{aligned}
& \dot{\xi}_{1}=\xi_{2} \\
& \dot{\xi}_{2}=-\sqrt{\frac{g}{2}}\left(\frac{\left(v-\sqrt{2 g h_{1}}-v q\right)}{\sqrt{h_{1}}}+\frac{\left(v q-\sqrt{2 g h_{2}}\right)}{\sqrt{h_{2}}}\right) \\
& y=\xi_{1}
\end{aligned}
$$

where

$$
\begin{aligned}
& h_{1}=\frac{1}{4}\left(\frac{v-\xi_{2}}{\sqrt{2 g}}+\mu \sqrt{2 \xi_{1}-\frac{\left(v-\xi_{2}\right)^{2}}{2 g}}\right)^{2}, \\
& h_{2}=\frac{1}{4}\left(\frac{v-\xi_{2}}{\sqrt{2 g}}-\mu \sqrt{2 \xi_{1}-\frac{\left(v-\xi_{2}\right)^{2}}{2 g}}\right)^{2} .
\end{aligned}
$$

Using this state transformation, one has to choose either the trajectories are in the subspaces $S_{O}^{+}=\left\{\left(h_{1}, h_{2}\right): h_{1}>h_{2}\right\}$ or $S_{O}^{-}=\left\{\left(h_{1}, h_{2}\right): h_{1}<h_{2}\right\}$, where $S_{O}$ is the boundary between both of them. Thus, $\mu=1$ if the state is in $S_{O}^{+}$and $\mu=-1$ if the state is in $S_{O}^{-}$. In both cases given in Fig. 6 and Fig. 7, the system behavior is principally or exclusively in the subspace $h_{1}>h_{2}$. Then the appropriate change of coordinates is $\mu=1$. This highlights the fact that the diffeomorphism is only a local

${ }^{8}$ Considering a system of the following form:

$$
\begin{aligned}
\dot{x} & =f(x)+g(x) q \\
y & =h(x)
\end{aligned}
$$

the observability matching condition with respect to $q$ is satisfied if $L_{g} h=\ldots=L_{g}^{n-2} h=0$ 
transformation and in this case the validity domains are strongly related to $S_{O}$.

Regarding the particular properties of the hybrid dynamical system (observability matching condition and regularly locally weakly observability) and taking advantage of the basic normal form (24), a higher order sliding mode observer [9, 16, 27] only made here of one super twisting algorithm step because the knowledge of the discrete state $q$ is not required. The proposed observer is the following:

$$
\begin{aligned}
\dot{\xi}_{1} & =\hat{\xi}_{2}+\lambda\left|y-\hat{\xi}_{1}\right|^{\frac{1}{2}} \operatorname{sign}\left(y-\hat{\xi}_{1}\right) \\
\dot{\xi}_{2} & =\alpha \operatorname{sign}\left(y-\hat{\xi}_{1}\right) \\
\hat{y} & =\hat{\xi}_{1}
\end{aligned}
$$

Setting $e_{1}=\xi_{1}-\hat{\xi}_{1}$ and $e_{2}=\xi_{2}-\hat{\xi}_{2}$, the observation error dynamics is:

$$
\begin{aligned}
& \dot{e}_{1}=e_{2}-\lambda\left|e_{1}\right|^{\frac{1}{2}} \operatorname{sign}\left(e_{1}\right) \\
& \dot{e}_{2}=\dot{\xi}_{2}-\alpha \operatorname{sign}\left(e_{1}\right)
\end{aligned}
$$

which is stable for appropriate values of $\alpha$ and $\lambda$ [27, 32]. In the given simulations, $\lambda=5, \alpha=40$ and a computation step equal to $10^{-5} s$ is used with the solver ode 5 of Matlab.

Case 1 (Fig. 6) exhibits a chattering Zeno phenomena $\left(I_{\min }=0\right.$ when the state behavior reaches the sliding manifold $h_{1}=2 h_{2}$ approximatively at time $t=0.2 s$ ) and the system slides on the switching manifold until $h_{1} \simeq 2.5$ and $h_{2} \simeq 1.25$ (see the green trajectory in Fig. 8 and Fig 9). Note that the Zeno time $t_{\infty}$ in this case is not unique, because during all the period the system slides on the switching manifold, there is an infinite number of commutations in every small time interval. The fact that the Zeno behavior is not restricted to a particular time instant $t_{\infty}$, but appears during a finite or infinite time interval is characteristic of a sliding mode behavior. Here, when the system has reached the switched manifold given by $h_{1}=2 h_{2}$, the system never leaves the constrained manifold (it is an invariant one).

The system behavior (in green) and the observer behavior (in red) are given in Fig. 8 and Fig. 9 for an appropriate choice of coordinate and a wrong choice of coordinate, respectively.

The observation errors in original coordinates are given in Fig 10. As the original behavior does not cross $S_{O}$ (in blue in Fig. 8 and Fig. 9), the observer (25) converges and it is not at all influenced by the Zeno behavior of the observed system (even if it is a chattering Zeno which slides on the switching manifold).

Case 2 (Fig. 7) exhibits a genuinely Zeno phenomena. Roughly speaking, the system switches more and more quickly and infinitely until the time $t_{\infty} \simeq 1.6$ which corresponds to the time when the state is equal to the desired one ( $h_{1}=4$ and $\left.h_{2}=1.5\right)$. After that, there is still life [2] (see the green trajectory in Fig. 12). The system trajectories in the phase plane are given in Fig. 11 and Fig. 12 with an appropriate and a wrong choice of coordinate, respectively. Life after Zeno is due to the fact that the input $v$ is strictly smaller than $v_{1}+v_{2}$ (the desired point is semi attractive, in fact the state behavior reaches $\left(h_{1}=4, h_{2}=1.5\right)$ only if the initial conditions 


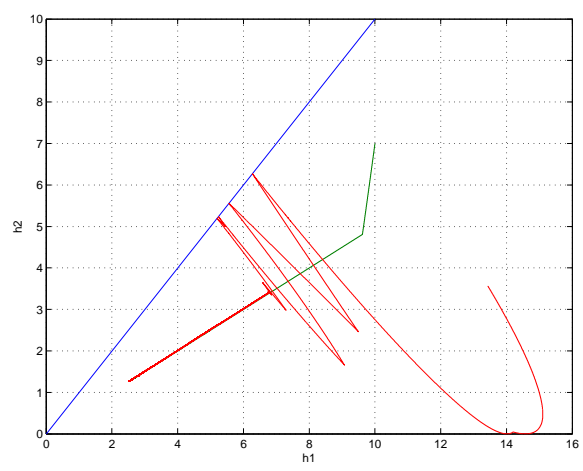

Fig. 8 The phase plane with an appropriate choice of coordinate.

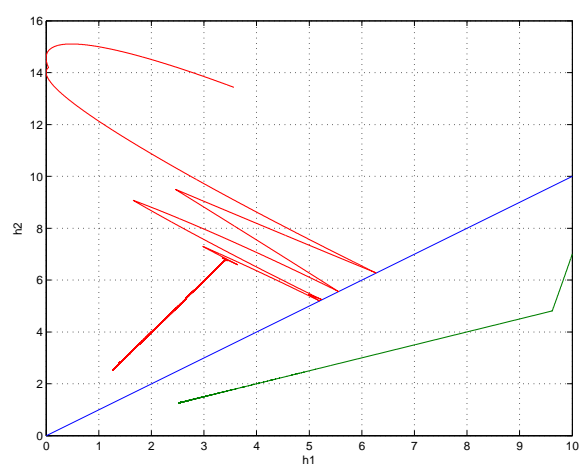

Fig. 9 he phase plane with a wrong choice of coordinate.

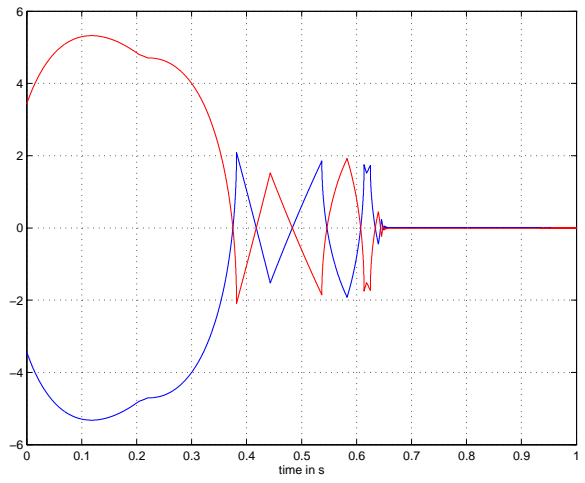

Fig. 10 The observation errors in the original coordinates. 
verify $h_{1}+h_{2} \gg 5.5$ ). Since the original system trajectory (in green) crosses the observability singularity set $S_{O}$, the observer trajectory diverges when the trajectory is in the opposite subspace. Moreover, when the system trajectory is close to the observability singularity set $S_{O}$, the observation errors increase even if the coordinates choice is correct.

The observation errors in the canonical coordinates $\xi$ are given in Fig. $13\left(e_{1}=\right.$ $\xi_{1}-\hat{\xi}_{1}$ in blue and $e_{2}=\dot{\xi}_{1}-\dot{\xi}_{1}$ in red). The observation errors are shown only during the first second, because the higher order sliding mode observer (25) converges in a finite time $\simeq 0.1 s$ and is not substantially perturbed by the $S_{O}$ crossing or by the Zeno behavior of the original system. The observation errors in the original coordinates $\left(h_{1}, h_{2}\right)$ are given in Fig. 14 and it is clear, for such coordinates, that the error dynamics are strongly influenced by the crossing of $S_{O}$. The Zeno time is $t_{\infty} \simeq 1.6 \mathrm{~s}$ (when $h_{1}=4$ and $h_{2}=1.5$ ). Then, the system converges to a limit cycle and the observer works well in both coordinates (original coordinates with appropriate choice and canonical coordinates) because the limit cycle does not cross $S_{O}$.

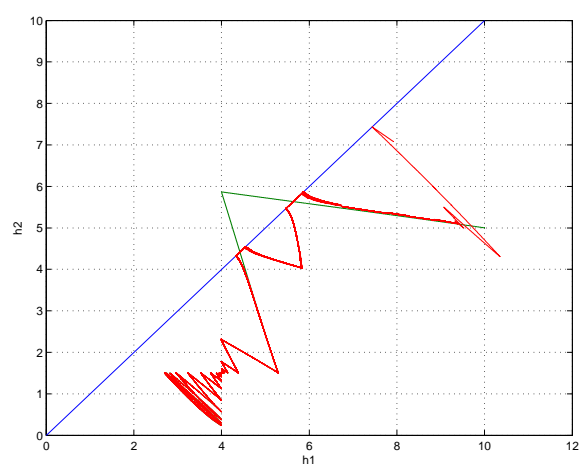

Fig. 11 The phase plane with an appropriate choice of coordinate.

\section{References}

1. A. Ames, A. Abate, S. Sastry. "Sufficient Conditions for the Existence of Zeno Behavior in Nonlinear Hybrid Systems via Constant Approximations". 44th IEEE CDC and ECC, 40334038, 2005.

2. A. Ames, H. Zheng, R. Gregg, S. Sastry, Is there life after Zeno? Taking executions past the breaking (zeno) point, IEEE ACC, Mineapolis, 2006

3. J.-P. Barbot, T. Boukhobza, M. Djemaï, Sliding mode observer for triangular input form, IEEE Conf. Decision Control, Kobe, 1996.

4. G. Besançon, Q. Zhang, H. Hammouri, High-Gain Observer based State and Parameter Estimation in Nonlinear System, 6th IFAC symposium on Nonlinear Control Systems, 2004.

5. D. Boutat, A. Benali and J.-P. Barbot, About the observability of piecewise dyamical system, 6th IFAC symposium on Nonlinear Control Systems, 2004. 


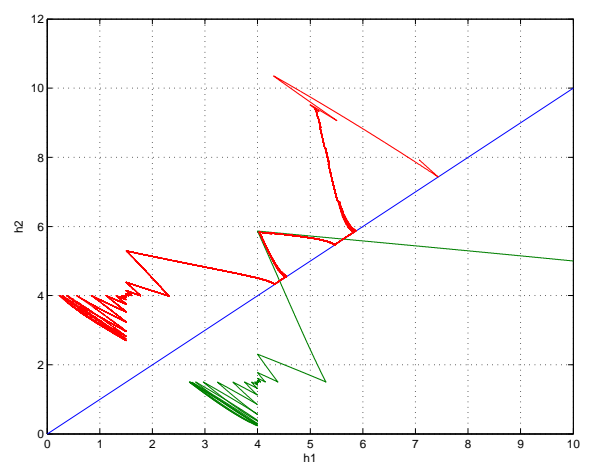

Fig. 12 The phase plane with a wrong choice of coordinate.

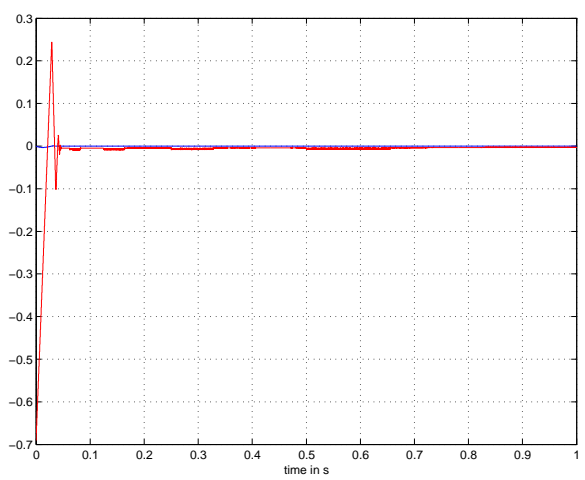

Fig. 13 The observation errors in the canonical coordinates.

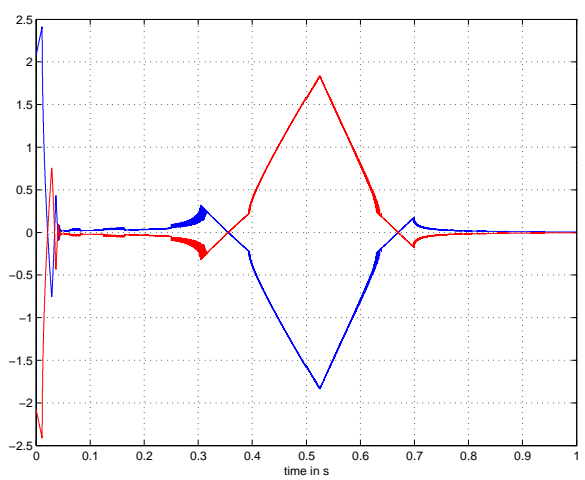

Fig. 14 The observation error in the original coordinates.

6. B. Brogliato, Nonsmooth Impact Mechanics: Models, Dynamics and Control, Lecture Notes in Control and Information Sciences 220, Springer-Verlag, 1996. 
7. K. Busawon and J. de Leon Morales, An observer design for uniformly observable nonlinear systems, Int. J. of Control, Vol. 73, No. 15, pp. 1357-1381. 2000

8. M. Cichon, I. Kubiaczyk and A. Sikorska, The Henstock-Kurweil-Pettis integral and exsitence theorems for the Cauchy problem, Czeckoslovak Mathematical Journal, Vol 54, pp 279-289, 2004.

9. J. Davila, L. Fridman, A. Pisano, E. Usai, Finite-time state observation for non-linear uncertain systems via higher-order sliding modes, International Journal of Control, Vol 8, 1564 1574, 2009.

10. S. De Fogio, The NV-Integrals, AMS Special Session on Nonabsolute Integration, Toronto, 2000.

11. S. Diop, M. Fliess, On nonlinear observability, Proc. $1^{\text {st }}$ Europ. Control Conf., Hermès, pp. 152-157, 1991.

12. S. Drakunov and V. Utkin, Sliding mode observer: Tutorial, in Proc. of the 34th IEEE CDC95, 1995.

13. S. V. Emelyanov and S. K. Korovin, Applying the principle of control by deviation to extend the set of possible feedback types, Soviet Physics, Doklady, Vol 26, No. 6, pp. 562-564, 1981.

14. A.F. Filippov, Classical solutions of differential equations with multi-valued right-hand sides, SIAM J. Control Vol 5 (1967), pp. 609-621.

15. A. F. Filippov, Differential Equations with Discontinuous Righthand sides, Kluwer Academic Publishers, 1988.

16. T. Floquet, J.-P. Barbot, Super twisting algorithm-based step-by-step sliding mode observers for nonlinear systems with unknown inputs, International Journal of Systems Science, Vol. 38, No 10, pp 803-815, 2007.

17. L. Fridman and A. Levant, Higher order sliding modes as the natural phenomenon in control theory, In Robust Control via Variable Structure and Lyapunov Techniques, Springer-Verlag, No 217, pp 107-133,1996.

18. R. Henstock, The General Theory of Integration, Oxford Mathematical Monographs, Clarendon Press, Oxford 1991.

19. R. Hermann, A. J. Krener, Nonlinear controllability and observability, IEEE Transactions on Automatic Control, Vol. 22, pp. 728-740, 1977.

20. M. Heymann, F. Lin, G. Meyer and S. Resmerita, Analysis of Zeno behaviours in hybrid systems, In Proc. of 41th IEEE CDC, Las Vegas, 2002.

21. K. H. Johansson, J. Lygeros, S. Sastry and M. Egerstedt, Simulation of zeno hybrid automata, Proc. 38th Conference on Decision and Control, Vol. 4, pp. 3538-3543, 1999.

22. W. Kang, J-P Barbot and L. Xu, On the observability of nonlinear and switched system, in press, in the book dedicated to Dr Wijesuriya P. Dayawansa, Springer, 2010.

23. Goebel R, Hespanha J, Teel A R, Cai C, Sanfelice R (2004) Hybrid systems: generalized solutions and robust stability. Proceedings of IFAC, Nolcos.

24. R. Goebel, R. Sanfelice R and A. Teel, Hybrid Dynamical systems, IEEE Control Systems Magazine, Vol. 29, No 2, pp. 28-93, 2009.

25. Leonov G, H. Nijmeijer, A. Pogromsky and A. Fradkov, Dynamics and Control of Hybrid Mecanical Systems, World Scientific Series on Nonlinear Science, Serie B, Vol 14, 2010.

26. A. Levant, Sliding order and sliding accuracy in sliding mode control, Int. J. of Control, Vol. 58, No. 6, pp. 1253-1253, 1993.

27. A. Levant, Robust Extract Differentiation via Sliding mode Technique, Automatica, Vol. 34, No. 3, pp. 379-384, 1998.

28. Lygeros J, Johansson H K, Simić S N, Zhang J, Sastry S S, Dynamical Properies of Hybrid Automata. IEEE Trans. on Autom. Control, Vol. 48, No.1, pp. 2-17, 2003.

29. M. Mboup, C. Join, M. Fliess, A revised look at numerical differentiation with an application to nonlinear feedback control, The $15^{\text {th }}$ Mediterrean Conference on Control and Automation, 2007.

30. M. Mboup, Parameter estimation via differential algebra and operational calculus, Rapport de recherche, http://hal.inria.fr/inria-00138294/fr, 2007. 
31. W. Perruquetti and J.-P. Barbot, Sliding Mode Control in Engineering, Ed. Marcel Dekker, 2002.

32. Saadaoui H., N. Manamani, M. Djemai, J.P. Barbot, and T. Floquet, Exact differentiation and Sliding mode observer for switched Lagrangian systems, in Nonlinear Analysis : Theory, Methods \& Applications, Vol. 65, No 5, pp. 1050-1069, 2006.

33. E. Talvila, Estimates of the remainder in Taylor's theorem using the Henstock, Czeckoslovak Mathematical Journal, Vol.55, pp. 993-940, 2005.

34. L. Yu, J.-P. Barbot, D. Boutat, D. Benmerzouk. Observability Normal Forms for a Class of Switched Systems with Zeno Phenomena, IEEE ACC, 2009. 